
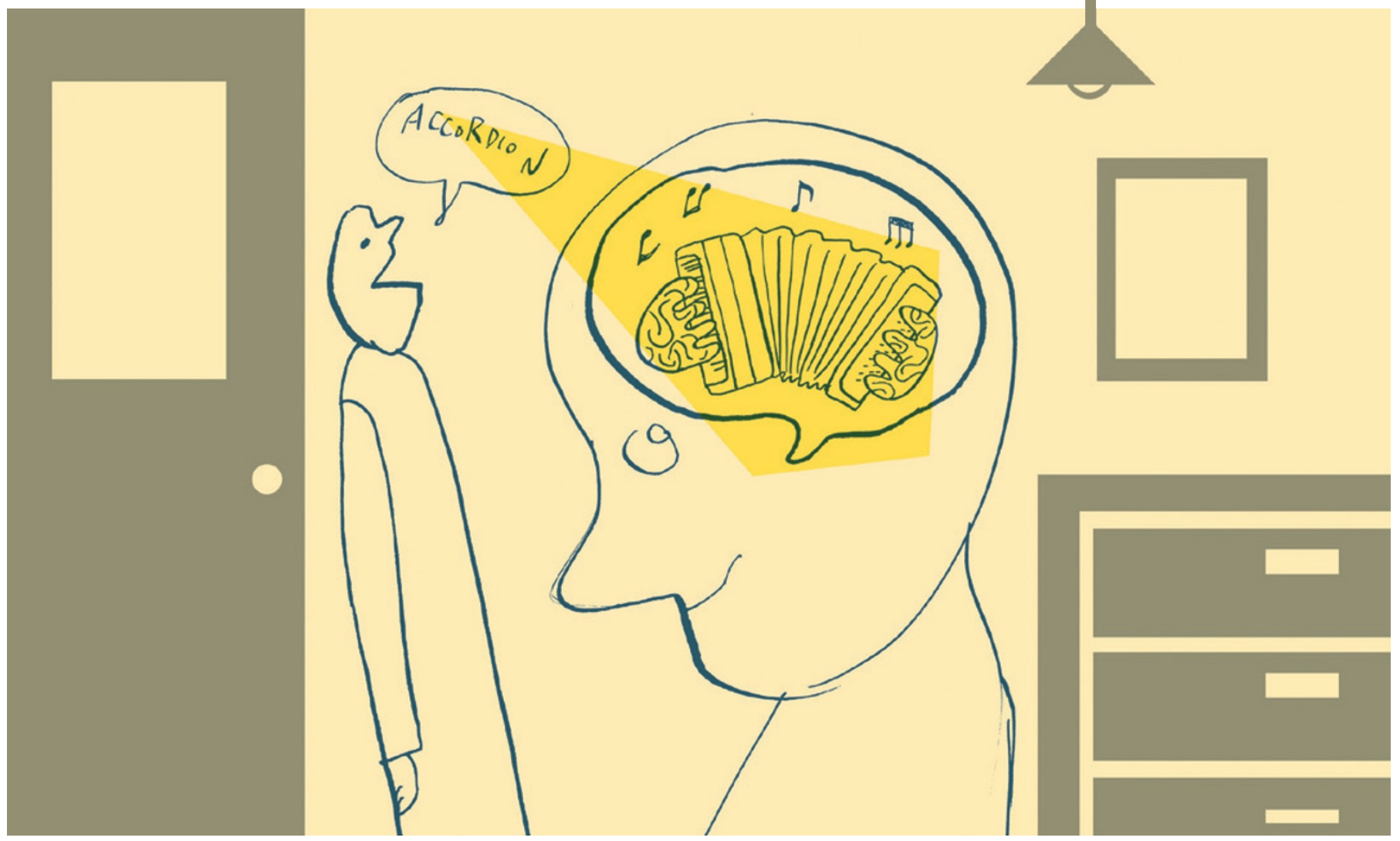

\title{
Found in translation
}

\section{Charles Fernyhough enjoys a bold exploration of how the mind extracts meaning from what we read or hear.}

$\mathrm{Y}$ ou are doing something quite remarkable. As you read these words, you are taking abstract symbols from the page or screen and extracting meaning from them. They are no longer mere squiggles of ink or pixels - or, in the case of spoken words, patterns of sound. You know what they refer to. Quite how the mind pulls off this nifty trick has troubled philosophers and cognitive scientists for as long as they have been thinking about language.

A prominent view within cognitive science is that linguistic terms are converted into signs or 'tokens' in a 'language of thought', sometimes known as Mentalese. These tokens correspond to the relevant entities in the world. When you read the word 'accordion', for example, a Mentalese token is activated, which allows you to have thoughts

$\rightarrow$ NATURE.COM

For more on linguistics, see: go.nature.com/isfbka about a noisy musical instrument played by squeezing. In his impressive debut, Louder Than Words, cognitive scientist Benjamin Bergen tries to persuade us of an alternative view: that we understand language through a process of embodied simulation. Bergen supports this view by reviewing around 200 scientific studies, by his count, from several teams that have been converging on this model during the past couple of decades.

According to Bergen's hypothesis, you

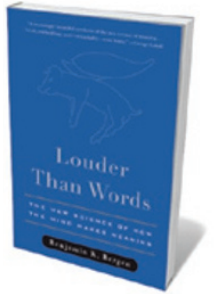

Louder Than Words: The New Science of How the Mind Makes Meaning BY BENJAMIN K. BERGEN Basic Books: 2012 312 pp.£18.99, $\$ 27.99$ understand the meaning of a word through the mental recreation of what it would be like to experience the thing being described. So when you hear the word 'accordion', the visual areas of your brain generate an image of an accordion. When you hear the verb 'squeeze', your motor system rehearses the firing that would achieve a squeeze, without going so far as to send the corresponding commands to your muscles.

Much of Bergen's evidence for this account relies on different interference effects, such as the trusty "action-sentence compatibility effect". For example, subjects are asked to read a sentence describing an action (such holding a marble with a closed fist) while simultaneously pressing a button in a way (such as with a flat palm) that is physically distinct from the described action. The mismatch between the described and performed actions slows language processing, suggesting that the comprehension of action-related language shares cognitive and neural resources with the real-life performance of those actions.

An obvious question is how Bergen's system deals with abstracts. Bergen reasons that much of our language about abstract concepts actually relies on concrete metaphors, meaning that both types of language can be underpinned by the same kinds of simulation. For 
example, studies show that when you read the phrase 'grasp a concept', you comprehend it faster if you have just performed a grasping action with your hand. This theme of complex cognitive abilities being bootstrapped off evolutionarily more ancient systems makes good biological sense. Bergen's view of the language system as a repurposed machine, building on more primitive capacities for perception and action, is one of the attractions of his argument.

But for those familiar with the philosophy and cognitive science of language, there are plenty of unanswered questions in Louder Than Words. Explaining how language performs its many functions is notoriously difficult, and the question of how a mental event can ever be 'about' an element of the real world remains a tricky philosophical issue. One problem is that Mentalese isn't the only game in town. You can have an account of meaning based on neural representations of conceptual knowledge that doesn't posit a language of thought. As a result, Louder Than Words sometimes has the feel of an assault on a straw man.

Bergen's treatment of language relies heavily on the 'conduit metaphor', by which one person's thoughts are packaged in words and sentences and unpacked by the listener or reader, rather than shaping and being shaped by a complex patterning of social exchanges. Bergen's theory lacks a developmental perspective: how do babies, for example, get started with word meanings? The model is also unclear on the role of consciousness. We are told that simulation can be unconscious, but elsewhere it is proposed to involve the "creation of mental experiences", presupposing that those experiences are conscious - because, by definition, if you experience something you must be conscious of it.

Bergen ends with a tricky question about the functional significance of mental simulation. Simulation happens when people process language, but does it actually achieve anything? His disarmingly honest conclusion, which dials back on a rather hyperbolic tone at the outset, is that simulation is ultimately neither necessary nor sufficient. He concedes that other forms of language understanding that don't involve embodied simulation are also important.

Bergen admits that his science is still in its infancy, and he sets out his account with enthusiasm, energy and some delightful touches of humour. If you want an engaging, well-informed tour of how cognitive science approaches the problem of meaning, you stand to learn a great deal from this book.

Charles Fernyhough is a reader in psychology at Durham University, UK, and the author of Pieces of Light, a book on autobiographical memory.

e-mail:c.p.fernyhough@durham.ac.uk

\section{Books in brief}

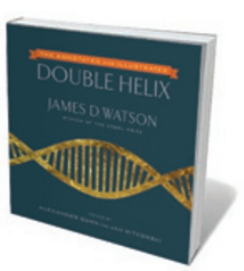

The Annotated and Illustrated Double Helix

James D. Watson, Alexander Gann and Jan Witkowski

SIMON \& SCHUSTER 368 pp. £19.99, \$30 (2012)

Few tales of modern science thrill as much as the race to discover DNA's double-helical structure. Fifty years after James Watson, Francis Crick and Maurice Wilkins won a Nobel prize for their sprint to the finish, Alexander Gann and Jan Witkowski have crafted a new edition of Watson's behind-the-scenes account, The Double Helix (1968). Annotated to clear up abiding mysteries; adorned with lab notes, sketches and photos; and beefed up with extras by Rosalind Franklin and other major players, this is a sampler of rare treats.

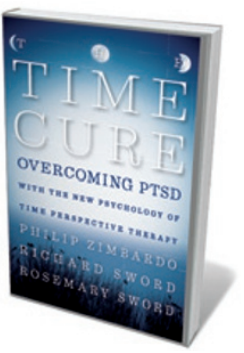

The Time Cure: Overcoming PTSD with the New Psychology of Time Perspective Therapy

Philip Zimbardo, Richard Sword and Rosemary Sword JOSSEY-BASS 336 pp. £17.99 (2012)

Psychologist Philip Zimbardo has long probed the nature of trauma — notably in his 1971 Stanford prison study — and how orientation towards the past or future affects mental well-being. Now, with therapists Richard and Rosemary Sword, he suggests these findings can guide treatment for people with post-traumatic stress disorder, who suffer harrowing flashbacks. A treatment plan (being tested by the US military), quantitative data and case studies are on offer.

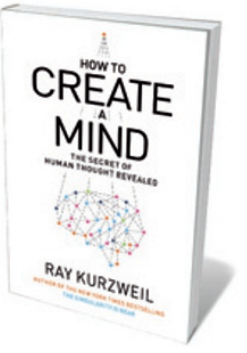

How to Create a Mind: The Secret of Human Thought Revealed Ray Kurzweil VIKING Books 352 pp. \$27.95 (2012)

In The Singularity Is Near (Viking, 2005), Ray Kurzweil imagined a near future in which medical nanotechnology would allow us to decant copies of our brains into hyper-intelligent machines - and effectively live forever. Now the bestselling futurist and pioneering inventor explores a prime arena for today's big science: reverse engineering the brain. Using the brain's pattern-recognition capacity as a springboard, Kurzweil leaps from the physical brain and the processes of creativity to the debatable idea that, given the correct software, digital entities are effectively conscious.

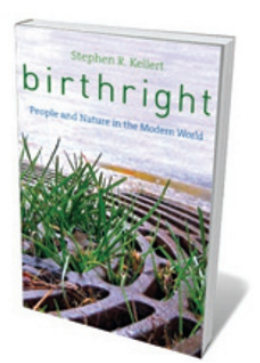

Birthright: People and Nature in the Modern World

Stephen R. Kellert YALE UNIVERSITY PRESS 264 pp. \$32.50 (2012)

With his sometime-collaborator E. O. Wilson, social ecologist Stephen Kellert has asserted that biophilia — affinity for nature - is central to health, emotional well-being and much more. Here, Kellert challenges our "adversarial" approach to nature with an exploration of eight ways in which we derive meaning from it, from attraction to exploitation. He argues that, even in cities, natural complexity and dynamism are central to children's cognitive development, as they recognize, identify and evaluate rocks, clouds, trees and insects. This is a nuanced analysis punctuated with insightful personal narratives.

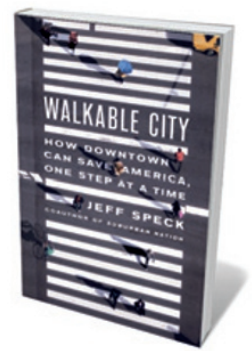

Walkable City: How Downtown Can Save America, One Step at a Time

Jeff Speck FARRAR, StRAuS AND GIROUx 320 pp. \$27 (2012)

Vast intersections, pin-thin pavements, kilometres of concrete: many working with scores of mayors, concluded that urban liveability demands walkability. He identifies the benefits of well-designed density, such as increased physical fitness, lower fuel use and higher productivity. His 'Ten Steps of Walkability', from well-shaped spaces to curbs on cars, are a blueprint for reclaiming downtown America. US city centres were built for cars, not feet. City planner Jeff Speck, 